\title{
Super-resolution imaging technique based on a LCoS display: Increase of CCD resolution limit
}

\section{Técnica de súper-resolución de imágenes basada en el uso de una pantalla LCoS: incremento del límite resolutivo impuesto por la CCD}

\author{
M. Sohail(1), A. $\operatorname{Lizana}^{(2, *)}$, J. Campos $(2, S)$ \\ 1. Department of Physics and Applied Mathematics, Pakistan Institute of Engineering and Applied Sciences, 45650 \\ Islamabad, Pakistan. \\ 2. Departamento de Física. Universitat Autònoma de Barcelona, 08193, Bellaterra, Spain. \\ ${ }^{*}$ Email: angel.lizana@uab.es S: miembro de SEDOPTICA / SEDOPTICA member \\ Received / Recibido: 03/04/2013. Revised / Revisado: 30/08/2013. Accepted / Aceptado: 03/09/2013. \\ DOI: http://dx.doi.org/10.7149/OPA.46.3.223
}

\begin{abstract}
:
The resolution of any imaging system is limited by many factors, as by the diffraction resolution limit of the system or by resolution limitations related to the geometry of the optical elements. One of the main geometrical limitations in optical systems is due to CCD cameras used for the image acquisition. In particular, pixel size, shape and pixel pitch of the used CCD camera always impose a limit in resolution. This paper faces the problem of the geometric super-resolution limitation, providing an approach free of mechanical movements, which helps to overcome the problems related to CCD pixels pitch. To this aim, a parallel aligned (PA) liquid crystal on silicon (LCoS) display is placed at the Fourier plane of a transparent object, and different linear phases are addressed to it. Afterwards, an image-forming optical system provides the final image of the object at the CCD camera plane. By addressing different linear phases to the LCoS display, object images with different sub-pixel displacements in 1-D are acquired by the CCD camera. Afterwards, all the shifted images are combined, leading to a final super-resolved image with larger dimension than the original object image. In addition, an inverse filtering process is also included into the proposed method, leading to certain extent, to a decrease of the blurring effect. The experimental comparison of the object images obtained with and without using the proposed technique provides the improvement, in terms of resolution, achieved by applying our technique.
\end{abstract}

Key words: Super-resolution, Geometric Super-resolution, Charge Coupled Device, Spatial Resolution, Parallel Aligned Liquid Crystal on Silicon (PA) LCoS.

\section{RESUMEN:}

La resolución de las imágenes formadas mediante el uso de un sistema óptico viene limitada por un cúmulo de factores, como el límite impuesto por la difracción producida en los diferentes elementos ópticos del sistema, o por la propia geometría de estos. Respeto a este último factor, una de las mayores causas que limitan la resolución de las imágenes está relacionada con factores geométricos propios de la cámara CCD utilizada para la captación de imágenes, como puede ser el tamaño de los píxeles, su forma o la distancia entre ellos. En este trabajo presentamos una nueva técnica de súperresolución de imágenes que permite mejorar el límite resolutivo impuesto por la cámara CCD. Para tal fin, utilizamos un sistema óptico libre de movimientos mecánicos gracias al uso de una pantalla de cristal líquido sobre silicio (LCoS). La transformada de Fourier de una escena se forma sobre el modulador, al que se le envían diferentes fases lineales. Una vez modificado el espectro del objeto, su imagen se forma sobre la cámara CCD. De este modo, diferentes fases lineales enviadas al modulador dan lugar a imágenes del objeto con diferentes desplazamientos sub-píxel sobre la cámara. Finalmente, todas las imágenes desplazadas se combinan para obtener una imagen de súperresolución, siendo ésta de mayor tamaño que las imágenes originales. Además, también hemos aplicado un post-procesado, basado en una técnica de deconvolución, sobre la imagen obtenida 
mediante la técnica de súper-resolución, permitiendo disminuir efectos no deseados relacionados con ruido añadido en las imágenes experimentales. Finalmente, se estudia la validez de la técnica de súper-resolución propuesta comparando las imágenes obtenidas con y sin usar la técnica.

Palabras clave: Super-Resolución, Cámara CCD, Resolución Espacial, Pantalla de Cristal Líquido LCoS.

\section{REFERENCES AND LINKS / REFERENCIAS Y ENLACES}

[1]. W. Lukosz, "Optical systems with resolving powers exceeding the classical limits II", J. Opt. Soc. Am. 57, 932-941 (1967). DOI

[2]. Z. Zalevsky, D. Mendlovic, Optical Super Resolution, Springer (2002).

[3]. A. Shemer, D. Mendlovic, Z. Zalevsky, J. Garcia, P. G. Martinez, "Super resolving optical system with time multiplexing and computer decoding", Appl. Opt. 38, 7245-7251 (1999). DOI

[4]. Z. Zalevsky, J. Solomon, D. Mendlovic, "Geometrical super-resolution using code division multiplexing", Appl. Opt. 42, 32-40 (2005).

[5]. A. Borkowski, Z. Zalevsky, B. Javidi, "Geometrical superresolved imaging using non-periodic spatial masking", J. Opt. Soc. Am. A 26, 589-601 (2009). DOI

[6]. M. Sohail, A. A. Mudassar, "Geometric super-resolution by using an optical mask", Appl. Opt. 49, 30003005 (2010). DOI

[7]. A. Borkowski, Z. Zalevsky, E. Marom, B. Javidi, "Enhanced geometrical super-resolved imaging with moving binary random mask", J. Opt. Soc. Am. A 28, 566-575 (2011). DOI

[8]. M. Sohail, A. A. Mudassar, "Geometric super-resolution using an optical rectangular mask", Opt. Eng. 51, 013203 (2012). DOI

[9]. Z. Zalevsky, D. Mendlovic, E. Marom, "Special sensor masking for exceeding system geometrical resolving power", Opt. Eng. 39, 1936-1942 (2000). DOI

[10].A. Ashok, M. A. Neifeld, "Pseudorandom phase masks for superresolution imaging from subpixel shifting", Appl. Opt. 46, 2256-2268 (2007). DOI

[11]. I. U. Haq, A. A. Mudassar, "Geometric superresolution of a CCD pixel", Opt. Lett. 35, 2705-2707 (2010). DOI

[12]. J. W. Goodman, Introduction to Fourier Optics, McGraw-Hill (1996).

[13]. M. Ben-Ezra, A. Zomet, S. K. Nayar, "Video super-resolution using controlled subpixel detector shifts," IEEE T. Pattern Anal. 27, 977-987 (2005). DOI

[14].K. Yu, N. Park, D. Lee, O. Solgaard, "Superresolution digital image enhancement by subpixel image translation with a scanning micromirror", IEEE J. Sel. Top. Quant. 13, 304-311 (2007). DOI

[15].H. C. Lan, M. L. Wu, E. M. Yeatman, "Non-mechanical sub-pixel image shifter for acquiring superresolution digital images", Opt. Express 17, 22992-23002 (2009). DOI

[16]. J. C. Russ, The Image Processing Handbook, CRC Press, Taylor \& Francis Group (2011).

[17]. L. Lobato, A. Lizana, A. Márquez, I. Moreno, C. Iemmi, J. Campos, M.J. Yzuel, "Characterization of the anamorphic and spatial frequency dependent phenomena in Liquid Crystal on Silicon displays", J. Eur. Opt. Soc. - Rapid Pub. 6, 1100 (2010).

[18]. W. Vanderlinde, J. Caron,"Blind deconvolution of SEM images”, ISTFA 2007 Conference Proceedings from the 33rd International Symposium for Testing and Failure Analysis, pp. 97-102 (2008).

[19].Y. Shechtman, S. Gazit, A. Szameit, Y. C. Eldar, M. Segev, "Super-resolution and reconstruction of sparse images carried by incoherent light", Opt. Lett. 35, 1148-1150 (2010). DOI

\section{Introduction}

High resolution devices are needed for many purposes such as remote sensing applications or for medical purposes. If the output image taken by imaging device is the same as input object, then we say ideal super-resolution is achieved. In real systems, the resolution of the signal is degraded by many reasons, as for instance, 
environmental conditions or physical limitations in resolution related to the optical components as well as geometrical components.

Aperture sizes, imperfections and misalignments of optical components impose a resolution limits related to the Point Spread Function (PSF) of the optical system [1,2]. The complete elimination of these signal degradation is impossible but can be minimized to some extent [1-3]. Modern imaging systems also include Charge-Coupled Devices (CCD) that lead to a reduction in resolution caused by the geometrical properties of pixel array, e.g. pixels shape, pixel size and separation of the pixels array (pixel pitch) [3-5].

Resolution of the imaging sensor depends on the density of the sampling points (i.e. the number of the pixels per unit area) and on the pixel size and geometry. Many approaches have been reported in literature to cover blurring and aliasing problems [4-12], which are originated by these CCD geometric properties.

Higher resolution than the provided by the sensor pixel density can be achieved by reconstructing several sub-pixel displaced images. To generate image sub-pixel displacements, the use of mechanical elements, as mirrors, can be applied $[13,14]$. Nevertheless, to avoid mechanical errors related to mechanical controlled displacements, the use of birrefringent materials, as for instance birrefringent crystals, can be also applied [15]. However, in such way, only a fixed value for the sub-pixel displacement can be generated.

In this paper, we present a new experimental approach which helps to improve the resolution limitation imposed by CCD pixels pitch by means of different image sub-pixel displacements generated by means of a spatial light modulator (SLM). This new technique lead to superresolved images without the necessity of use any mechanical element in the set-up, providing a great flexibility to the system, in terms of displacements generation.

\section{LCoS display based super-resolved image technique}

\section{2.a. Description of the set-up}

The set-up proposed to achieve super-resolved images of a transparent object includes a reflective parallel aligned (PA) liquid crystal on silicon (LCoS) display spatial light modulator (SLM) model PLUTO from HoloEye, with aresolution of $1920 \times 1080$ pixels, a pixel pitch of $8 \mu \mathrm{m}$, and fill factor of $87 \%$. The whole system is illuminated by using a He-Ne laser $(633 \mathrm{~nm})$. Before the PA-LCoS display, a scene-illuminating system is placed. It is formed by a spatial filter (SF) plus a convergent lens, a linear polarizer (LP) and the convergent lens L1. First, the combination of the SF and the convergent lens allows the system to generate a cleaned-of-noise and collimated beam impinging the LP. It is placed with its transmissive axis oriented at 90 degrees of the laboratory vertical (i.e. at the same orientation of the liquid crystal molecules extraordinary axis). In this way, the PA-LCoS display operates in a phase-only configuration. Afterwards, the collimated beam exiting from the LP reaches the convergent lens L1 (focal length of $\mathrm{f} 1 \sim 20 \mathrm{~cm}$ ), leading to a convergent illumination of the object. At the focal plane of the lens L1, the Fourier spectrum of the object with a given scaled factor is formed. The scaled factor of the Fourier spectrum depends on the focal length of L1 and the distance between L1 and the PA-LCoS display. Thus, a proper relation of these two parameters must be selected to ensure a matching between the Fourier spectrum size and the PA-LCoS display dimensions. We are assuming that the optical system that forms the image is not the limiting factor. In this optical system the LCoS should be included. So, ideally, the band-pass of the LCoS should be equal to the rest of the optical system.

In addition, a non-polarizing beam splitter (B-S) is also included in the set-up, to steer the light reflected by the PA-LCoS display at the reflected set-up arm. At this stage, we address different linear phases to the PA-LCoS display to encode the object spectrum plane. At the reflected beam, the convergent lens L2 (focal length of $\mathrm{f} 2 \sim 20 \mathrm{~cm}$ ) is placed. Then, the encoded object spectrum at the PA-LCoS display plane is 


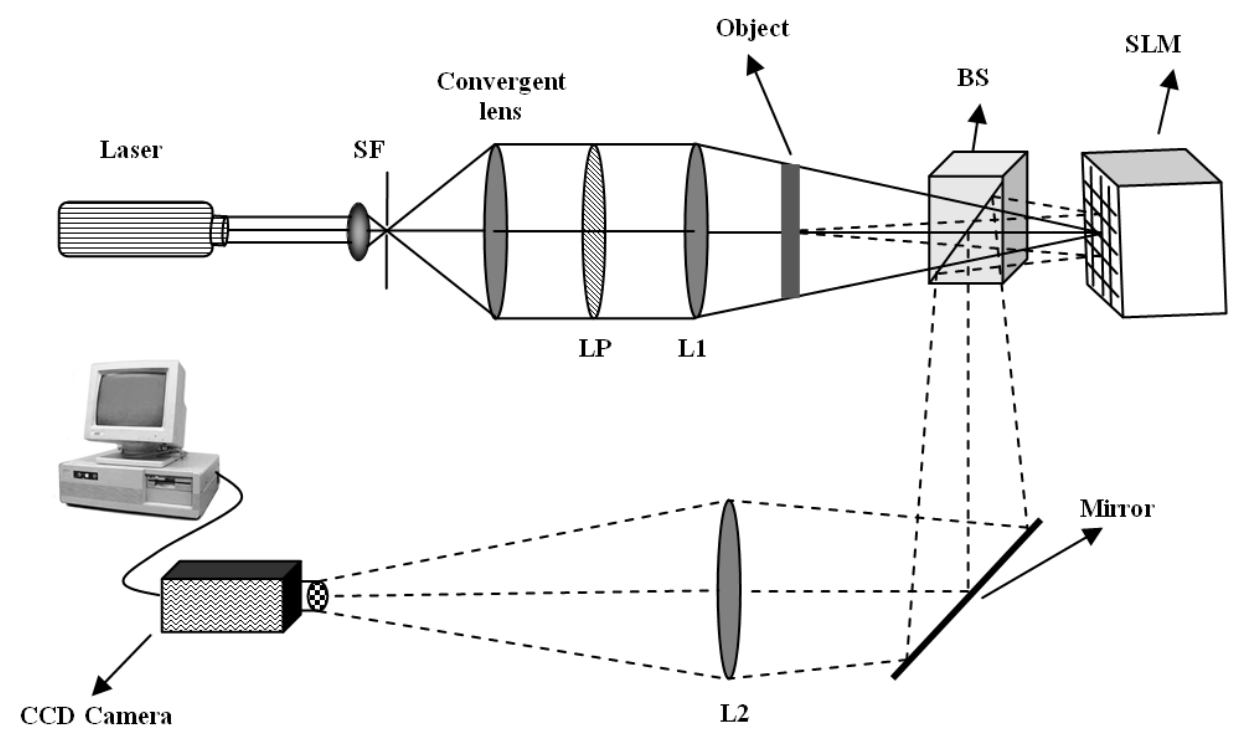

Fig. 1. Experimental set-up.

inverse Fourier transform by L2 which produce the image of the object at the imaging plane. Finally, the resulting image of the object is sampled by the CCD camera. The camera used in this work is a charge coupled device (CCD) model piA1000-60gm from Basler, with a resolution of $1000 \times 1000$ pixels and with a pixel pitch of $7.4 \mu \mathrm{m}$.

Finally, the PA-LCoS display is used to generate different linear phases at the Fourier spectrum plane of the object. Different encodedlinear phases lead to different shifts of the object image at the image plane, where the CCD camera is placed. At this stage, by controlling the value of the linear phase addressed to the LCoS display, the image is shifted by some fraction of the CCD pixel size, performing sub-pixel displacements in 2-D of the image object. Finally, all the images are combined leading to a final image with larger dimension than the original object.

Different linear phases addressed to the PALCoS display lead to different shifts of the object image at the CCD camera. The system is calibrated to determine the linear phase corresponding to a displacement of 1 pixel at the CCD camera in the $\mathrm{x}$ and $\mathrm{y}$ directions. By knowing this, it is immediate to determine the linear phase required to produce any displacement of the object image at the CCD camera. In this work, fractions of pixel size (i.e. sub-pixel displacements) are generated in 2-D. For every fraction $\left(1 / n_{x}\right) \cdot\left(1 / n_{y}\right)$ of the pixel size selected, $n_{x} \cdot n_{y}$ images are recorded to homogenously cover the whole pixel dimension. Finally all the displaced images are properly combined, obtaining the final super-resolved image.

\section{2.b. Simulated and experimental results}

First, the proposed super-resolved image technique is tested by conducting different simulations related to diverse sub-pixel displacements when an USAF chart is used as an object. The results are given in Fig. 2, where we provide a comparison between the original object image (i.e. the low resolution image, Fig. $2(a))$, and the final image obtained when performing our technique for different sub-pixel displacements, both in $\mathrm{x}$ and $\mathrm{y}$ directions: $1 / 2$, $1 / 4$ and $1 / 6$ of the pixel size (Fig. 2(b), 2(c) and 2 (d) respectively). Due to the increase in information provided by the different displaced images, a significant improvement in resolution is observed when comparing the low resolution image (Fig. 2(a)) with the object images related to different sub-pixel displacements (Fig. 2(b)(d)). This significant improvement is easily observed both in $x$ and $y$ directions (i.e. vertical and horizontal lines). Finally, we want to note 


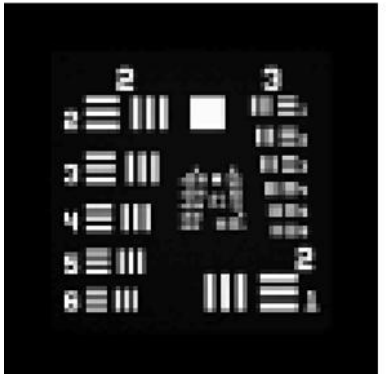

(a)

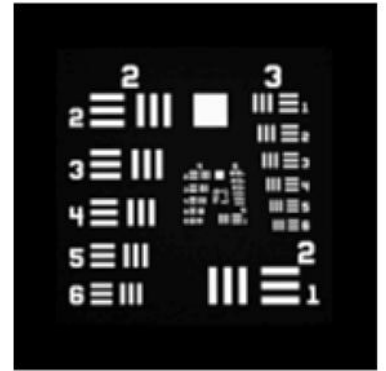

(b)

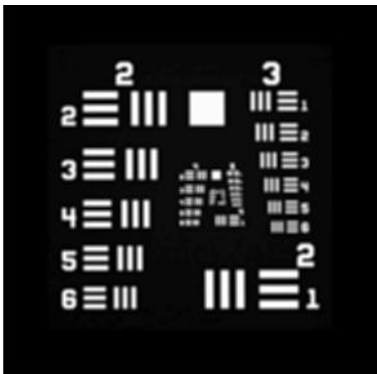

(c)

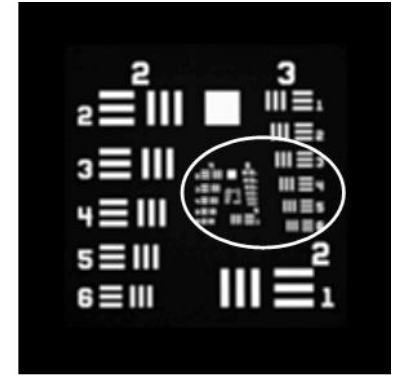

(d)

Fig.2. (a) Low resolution image; High resolved image for a sub-pixel displacement of: (b) 1/2, (c) 1/4 and (d) 1/6 of the pixel size.

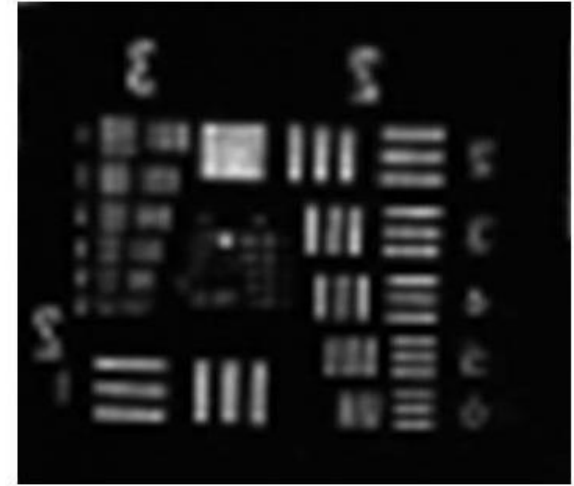

(a)

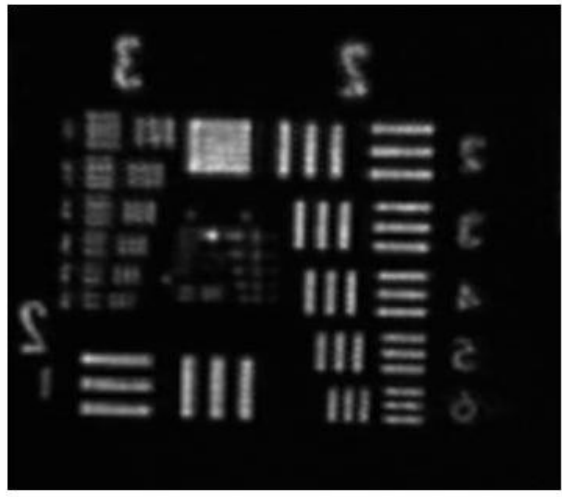

(b)

Fig. 3. (a) Experimental low resolution image; (b) High resolved image.

that resolution related to displacements of $1 / 4$ and $1 / 6$ of the pixel size, is approximately the same (Fig. 2(c) and 2(d)). Therefore, from that $1 / 4$ displacement value, no significant extra resolution improvement is achieved if continuing decreasing the sub-pixel displacement value. We think that this limitation is related to the blurring effect, originated by the intensity average conducted at the CDD pixels area [16].

Finally, the simulated results previously discussed are verified by performing experimental measurements, by using the set-up described in section 2.a. Figure 3 provide an experimental comparison between the original object image (Fig. 3(a)) and the final image obtained when experimentally performing our technique with displacements of $1 / 2$ pixel size both in $x$ and $y$ directions (Fig. 3(b)). A significant improvement in resolution is easily observed both in $x$ and $y$ directions (i.e. vertical and horizontal lines in Fig. 3(b)). We want to emphasize that a slightly greater improvement is reached in $x$-direction than in $y$-direction (see vertical and horizontal lines under the number 3). From a theoretical point of view, the improvement must be the same, but when experimentally implementing the technique, different factors have to be taken into account. First, the resolution of the PA-LCoS display used is of $1920 \times 1080$ pixels, and thus, larger for the $x$ direction. Moreover, the anamorphic phenomenon in LCoS display [17], which may degrade the efficiency of the holograms addressed to this device, has proved to be more significant for the $y$-direction than for the $x$ direction. Therefore, the combination of these two factors specific of LCoS displays may lead to smaller hologram efficiency in the $y$-direction than in the $x$-direction.

Smaller displacements (and so, larger dimension images) have been also tested, but the increase in resolution is no so evident when compared with results given in Fig. 3(b). We think that this is because in this situation image resolution is not limited by CCD pixel geometry 
but by the PSF of optical system and/or the blurring effect.

\section{Use of an inverse filtering process to reduce blurring effect}

\section{3.a. Deconvolution technique}

Note that all the acquired images suffer the spatial average due to the size of the pixel. This average can be seen as a low pass filter in the Fourier domain that can be partially compensated by applying an inverse filter, leading to a reduction of the blurring effect in the final images [16]. Intensity images provided by CCD cameras always present certain noise content related to different noise sources, as readout noise, thermal noise, dark current noise and photon noise, among others. If the noise content in the intensity image acquired is significant, it can greatly degrade inverse transforming processes applied to it, because the numerical precision may be exacerbate and overflow [18]. Therefore, to evaluate the possible improvement related to the use of an inverse filtering process in our method, we also apply a deconvolution process to the images provided by our CCD camera. In particular, we use the relation given in Eq. (1), deduced by conducting the convolution of the object image with the square function of the CCD pixel and by Fourier transforming the result:

$$
\tilde{\theta}(v)=\frac{\tilde{I}(v) H^{*}(v)}{|H(v)|^{2}+\sigma},
$$

where $\tilde{I}(v)$ is the Fourier transform of the intensity sampled by the CCD camera, $\tilde{\theta}(v)$ is the Fourier transform of the object and $H(v)$ is the $\operatorname{sinc}(v)$ function corresponding to the Fourier transform of a rectangle whose number of pixels is equal to the number of shifted images. Note that in Eq. (1) there is also included the

parameter $\sigma$. This parameter is imposed to be small and is included to avoid zeros at the denominator of Eq. (1) and not to enhance the noise in those zones where the signal is low in comparison with the noise. Finally, by inverse Fourier transforming Eq. (1), a filtered object image (i.e. removed to certain extent of blurring effect) is achieved.

\section{3.b. Results}

Simulated results of our super-resolution imaging technique are provided for the USAF chart scene in Fig. (4). In particular, we provide a comparison between the results obtained when applying our technique, without using the inverse filter described in section 3.a (images in first row in Fig. 4), and by using it (second row in Fig. 4). The analysis is conducted for three different sub-pixel displacements: $1 / 2,1 / 4$ and $1 / 6$ of the pixel size (Fig. $4(\mathrm{a}), 4(\mathrm{~b})$ and $4(\mathrm{c})$
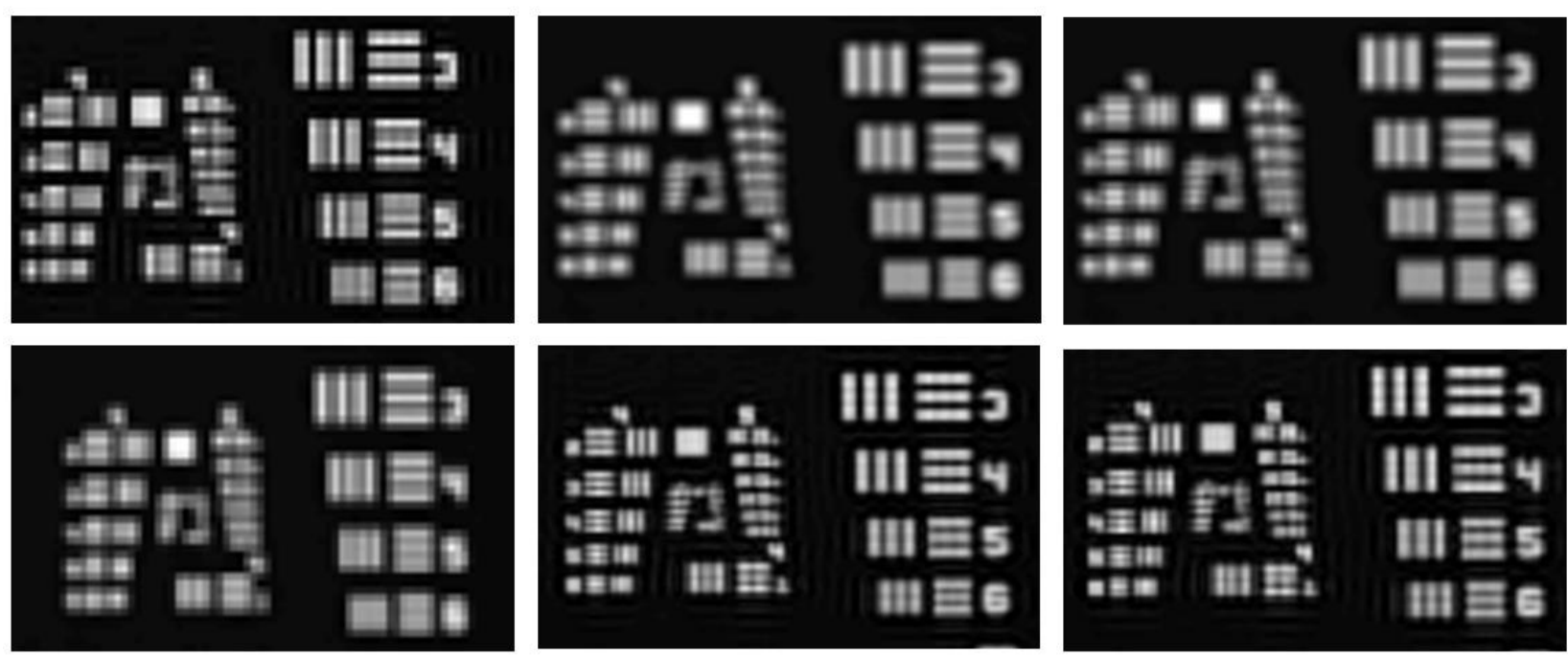

(a)

(b)

(c)

Fig. 4. Object image obtained by performing a displacement of: (a) $1 / 2$, (b) $1 / 4$ and (c) $1 / 6$ of the pixel size. The simulations are obtained without using (first row) and by using (second row) the inverse filter. 

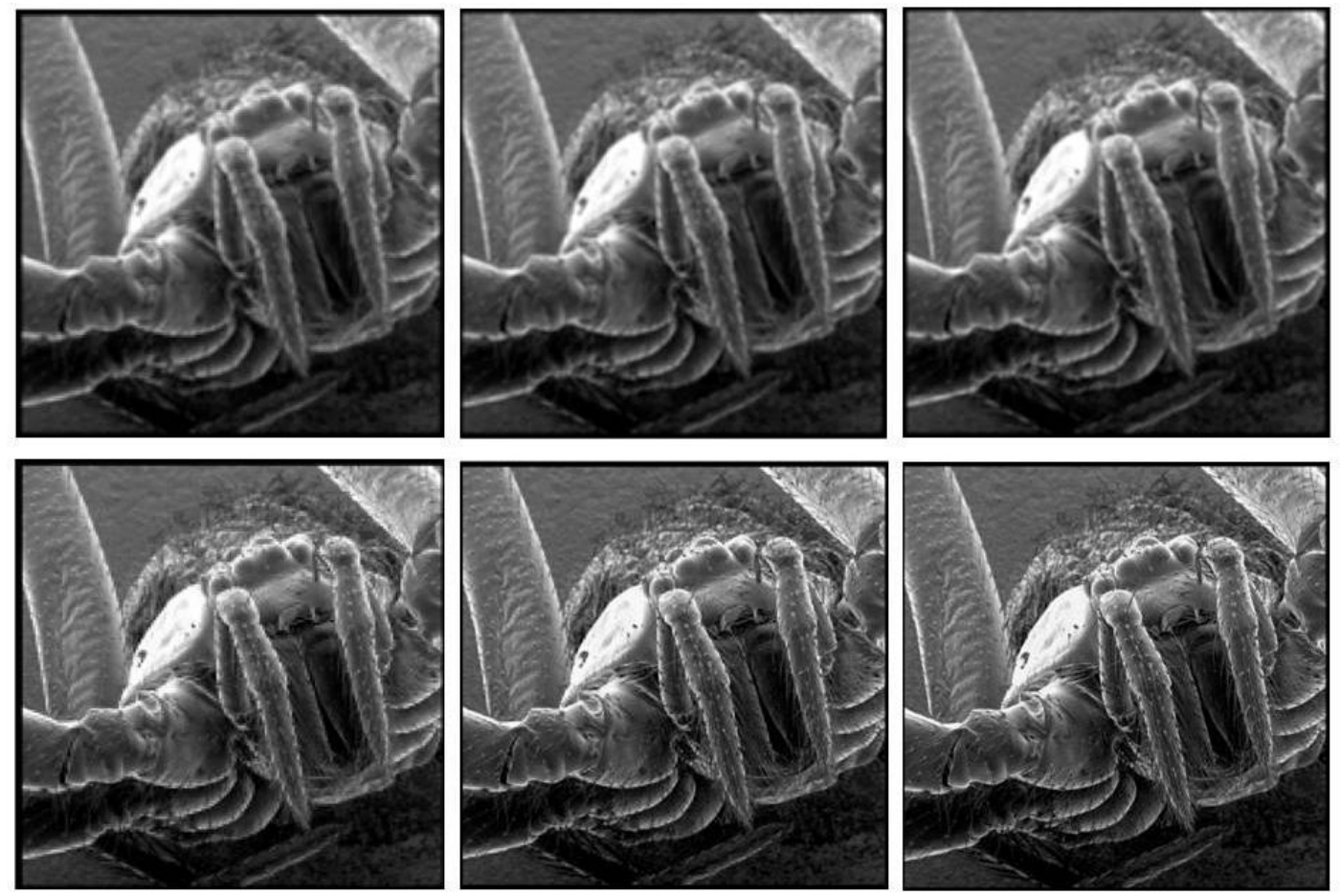

Fig. 5. Spider image obtained by performing a displacement of: (a) 1/2, (b) 1/4 and (c) $1 / 6$ of the pixel size. The simulations are obtained without using the inverse filter (first row) and by using it (second row).

respectively). In order to obtain better insight on the obtained results, the object region showing the limit in resolution of our provided technique (i.e. the higher frequencies in object we are able to resolve) is zoomed. In particular, the object region used to comparison is marked with a white line in Fig. 2(d).

As previously discussed in section 2, when image information extracted by using sub-pixel displacements is considered, an increase in the final image resolution is achieved (first row in Fig. 4). Moreover, by applying as well the inverse filter (images at the second row in Fig. 4), the increase in resolution is clearly larger than without applying it (first row in Fig. 4). This is because by applying the deconvolution technique, we are reducing to certain extent the image resolution limitation imposed by the blurring effect. Therefore, this result points out the improvement in terms of resolution of applying the inverse filtering process to the proposed technique.

Finally, to test the super-resolved image technique with different scenes, an extra experiment is also provided, this time by using as scene the image of a spider. Again, the simulations are conducted for different displacements and by applying the technique with and without using the deconvolution technique. The obtained results are shown in Fig. 5, providing the images obtained when applying our technique for sub-pixel displacements of $1 / 2,1 / 4$ and $1 / 6$ of the pixel size (Fig. 5(a), (b) and (c) respectively). In addition, a comparison between the spider images obtained without using the inverse filter (first row in Fig. 5), and by using it (second row in Fig. 5) is also provided. As in the case of the USAF chart scene (Fig. 4), we observe that the use of the inverse filter reduces the blurring effect, leading to an improvement of the final image resolution. In this sense, spider images in the second row of Fig. 5, obtained by applying the invers filter, clearly provide better image resolution than spider images in the first row, obtained without using it. In addition, by using smaller sub-pixel displacements, the resolution is also improved. For instance, image spider details, related to high frequencies, show better definition in Fig. 5(c) (1/6 of the pixel size displacements) than in Fig. 5 (a) $(1 / 2$ of the pixel size displacements). Therefore, results in Fig. 4 and 5 provide the significance of combine the 
inverse filter with the super-resolved image technique, leading to the best result in terms of image resolution.

\section{Conclusions}

Summarizing, this paper provides an experimental approach, based on a PA LCoS display technology, valid to improve resolution limitations imposed by the pixel pitch in CCD cameras. To properly use this technique, certain experimental conditions must be fulfilled, as the use of a coherent light source, of a spatial light modulator (SLM) and the implementation of a set-up including both a scene-illuminating system and an image-forming optical system (see Fig. 1). Different methods are nowadays able to achieve super-resolution imaging overcoming some of the initial restrictions in our technique, as for instance, in ref. [19], where super-resolution images are achieved by using incoherent illumination (for sparse light) or in [15], where a simplest set-up is proposed. However, the potential of our technique lies in the fact that by including a SLM in the system, any image scanning procedure is required. Therefore, the proposed technique not only completely avoids errors related to mechanical movements of optical elements in the system, but also provides a robust and flexible set-up, being potentially useful for a large number of applications, as for instance, those related to microscopy.
In particular, a PA-LCoS display is used to generate different linear phases on the Fourier spectrum of an object, leading to different displacements of the object image sampled by CCD camera. By means of the proper combination of the different shifted images, a super-resolved image of the object is obtained. In addition, an inverse filtering process is applied as well, enabling to decrease, to a certain extent, the blurring effect introduced due to the intensity average performed inside the pixels area. Simulated and experimental results are also provided in this work, showing the significant improvement in resolution of the images obtained with the proposed technique.

Finally, we want also to emphasize that the study presented in this work is performed for transparent samples, as the experimental set-up is devised for measurements in transmission. However, the set-up can readily be adapted to perform measurements in reflection, without neither imposing extra limitations to the current procedure nor detracting efficiency to the technique.

\section{Acknowledgements}

We acknowledge support from the Spanish Ministry of Science and Education and FEDER (FIS2009-13955-C02-01).

M. Sohail acknowledges Higher Education Commission (HEC) of Pakistan for funding the grant. 